\title{
Short Communication: Low Immune Activation Is Associated with Higher Frequencies of Central Memory T Cell Subset in a Cohort of Indian Long-Term Nonprogressors
}

\author{
Vandana Saxena,' Shubhangi Bichare,' Dharmendra Singh,' Manisha Ghate, Sheela Godbole, \\ Smita Kulkarni, ${ }^{4}$ Raman Gangakhedkar, Ramesh Paranjape, and Madhuri Thakar ${ }^{1}$
}

\begin{abstract}
Persistent immune activation in human immunodeficiency virus (HIV) infection is responsible for alterations in immune system such as activation, apoptosis, and reduced frequencies. Reduced immune activation is known to be associated with virus control. Limited information is available on the influence of pan-immune activation on memory responses. Hence, we compared the T cell activation and memory profile in HIV-infected individuals exhibiting disease control such as long-term nonprogressors (LTNPs) and progressors. The activated CD4 ${ }^{+}$and $\mathrm{CD}^{+} \mathrm{T}$ cells were significantly lower and the $\mathrm{CD} 4^{+}$and $\mathrm{CD} 8^{+}$central memory $\mathrm{T}$ cell phenotypes were significantly higher in the LTNPs compared to the progressors. In addition, we observed significant inverse association between the $\mathrm{T}$ cell activation and frequencies of central memory $\mathrm{T}$ cells. Our findings indicate that patients with absence of disease progression have preserved central memory $\mathrm{T}$ cell population associated with lesser immune activation.
\end{abstract}

Keywords: long-term nonprogressors, T cells, immune activation

$\mathbf{H}$ UMAN IMMUNODEFICIENCY VIRUS (HIV) induces persistent immune activation resulting in pan-activation of the immune system. This is characterized by activatory molecule expression on immune cells, including $\mathrm{T}$ and $\mathrm{B}$ cells, natural killer cells, and secretion of proinflammatory cytokines. ${ }^{1,2}$ The persistent immune activation in HIVinfected individuals is correlated with faster disease progression. ${ }^{3-5}$ However, limited information is available on its influence on the memory $\mathrm{T}$ cell compartment. One study has shown that immune activation alters the CD8 memory cell differentiation. ${ }^{6}$ Since long-term maintenance of memory is important for the generation of faster and efficient recall $\mathrm{T}$ cell responses and impaired CD4 central memory $\mathrm{T}$ cell response $\left(\mathrm{T}_{\mathrm{CM}}\right)$ was found to be associated with HIV disease progression, 7,8 it is important to understand the influence of immune activation on memory $\mathrm{T}$ cell compartment.

We used HIV-infected long-term nonprogressors (LTNPs) as a model for control over HIV disease progression. LTNPs represent a group of asymptomatic, HIV-infected individuals with stable CD4 count of $\geq 500$ cells $/ \mathrm{mm}^{3}$ for at least seven or more years of documented HIV infection in the absence of antiretroviral treatment (ART). ${ }^{9}$ LTNPs are shown to have a variable degree of immune activation in different studies. ${ }^{10-}$ ${ }^{12}$ It was reported that despite a high viral load, the lower immune activation and preserved immune responses result in long-term asymptomatic disease stage in patients of Amsterdam cohort, ${ }^{10}$ while chronic immune activation has been shown to be associated with progressive HIV. ${ }^{13,14}$ Furthermore, the persistent immune activation is shown to drive the $\mathrm{T}$ cell differentiation resulting in replicative senescence. ${ }^{15}$ Hence, we hypothesize that lower immune activation of $\mathrm{T}$ cells in LTNPs may ultimately result in better preservation of memory $\mathrm{T}$ cell population compared to the chronic progressors having a higher percentage of activated $\mathrm{T}$ cells. To test this hypothesis, we assessed the frequencies of activated T cells expressing HLA-DR and CD38 as a marker of immune activation, as well as the frequencies of central $\left(\mathrm{T}_{\mathrm{CM}}\right)$ and effector memory $\left(\mathrm{T}_{\mathrm{EM}}\right) \mathrm{T}$ cells in LTNPs.

Departments of ${ }^{1}$ Immunology and Serology, ${ }^{2}$ Clinical Sciences, ${ }^{3}$ Epidemiology and Biostatistics, and ${ }^{4}$ Virology, National AIDS Research Institute, MIDC, Pune, India. 
Thirty-one $(11 \mathrm{M} / 20 \mathrm{~F})$ LTNPs were recruited from the LTNPs cohort who are under regular follow-up at National AIDS Research Institute along with $21(11 \mathrm{M} / 10 \mathrm{~F})$ chronic progressors (ART-naive HIV-infected patients with CD4 count $<500$ cells $/ \mathrm{mm}^{3}$ ) attending the outpatient clinics of the institute. The median age was 38 years in both LTNPs (range 30-75 years) and chronic progressors (range 22-48 years). The median seropositivity period of LTNPs was 11 years (range 8-19 years). At the time of enrolment in this study, the median CD4 count was 630 and 300 cells $/ \mathrm{mm}^{3}$ in LTNPs and progressors, respectively. The median plasma viral load (pVL) was significantly lower $(5,321$ copies/ml; range 40$770,523)$ in LTNPs than that in progressors $(28,345$ copies/ $\mathrm{ml}$; range $1,065-1,456,931)(p=.012)$. The participants were not tested for M. tuberculosis infection; however, none of them presented with any signs or symptoms of tuberculosis disease. In addition, five HIV-seronegative healthy individuals (healthy controls; range $28-33$ years; $3 \mathrm{M} / 2 \mathrm{~F}$ ) with selfreported history of no fever in last 2 weeks and no major systemic disease were also enrolled for comparison. The study was approved by the Institutional Ethics Committee and prior written informed consent was obtained from all the participants.

The frozen peripheral blood mononuclear cells (PBMCs) from the study participants were revived as described previously ${ }^{16}$ and viability of the cells was assessed using trypan blue dye exclusion method. The samples showing $>90 \%$ viability and $>3 \times 10^{6}$ cell count were considered for further analysis. $1 \times 10^{6}$ PBMCs each were used to characterize activation status of $\mathrm{T}$ cells and determine frequencies of memory $\mathrm{CD}^{+}$and $\mathrm{CD}^{+} \mathrm{T}$ cells using multicolor flow cytometry. The following antibodies were used: anti-CD3PE-Texas-Red-A (Invitrogen), anti-CD4-APC-Cy7, anti-CD8PE-Cy7, anti-CD38-FITC, anti-HLA-DR-PerCP-Cy5.5, anti-CD45RA-APC, CD67L-PE, and CCR7-PE-Cy7 (all from BD Pharmingen). PBMCs were incubated with specific antibodies for $30 \mathrm{~min}$ in dark at RT, followed by washing with a wash buffer (phosphate-buffered saline $+0.5 \%$ fetal calf serum). The cells were fixed in 3\% formaldehyde, acquired, and analyzed on FACSAria (Becton Dickinson) using FlowJo software. A total of 50,000 gated events of lymphocytes were acquired. Appropriate cell and isotype controls were used for determination of CD38/HLA-DR/HLA-DR and $\mathrm{CD} 38$ coexpressing $\mathrm{CD} 4^{+}$and $\mathrm{CD}^{+} \mathrm{T}$ cells and to determine the frequencies of CD4 and CD8 central memory $\left(\mathrm{CCR}^{+} / \mathrm{CD} 2 \mathrm{~L}^{+}\right.$and $\left.\mathrm{CD}^{+} 5 \mathrm{RA}^{-}\right)$and effector memory $\left(\mathrm{CCR}^{-} / \mathrm{CD}^{2} 2 \mathrm{~L}^{-}\right.$and $\mathrm{CD}^{-} \mathrm{RA}^{-}$) population. The gating strategy to identify the different populations is shown in Supplementary Figure S1 (Supplementary Data are available online at www.liebertpub.com/aid). The GraphPad Prism version 4.0 software was used for statistical analysis and presentation. Kruskal-Wallis test with post hoc analysis was used to compare the differences in the levels of T cell activation markers (Fig. 1A). Nonparametric Mann-Whitney $U$ test was used to compare the differences in the frequencies of memory $\mathrm{T}$ cell phenotype between LTNPs and progressors, (Fig. 1F). Spearman test was used for correlation analyses. A $p$-value of $<.05$ was considered significant.

The progressors had a significantly higher expression of activation markers, HLA-DR, CD38, and HLA-DR+CD38 on CD4 and CD8 T cells compared to the healthy controls $(p<.05$; Fig. 1A). Progressors also had a higher expression of
HLA-DR and HLA-DR+CD38 on both $\mathrm{CD} 4^{+}$and $\mathrm{CD}^{+} \mathrm{T}$ cells compared to the LTNPs $(p<.001 ;$ Fig. 1A), but not CD38-expressing T cells ( $p>.05$; Fig. 1A). The LTNPs had similar frequencies of HLA-DR as well as HLA-DR+CD38coexpressing CD4 and CD8 T cells compared with healthy controls ( $p>.05$; Fig. 1A), except the expression of CD38, which was significantly higher in LTNPs than in healthy individuals $(p<.05$; Fig. 1A). We also determined whether the immune activation had any association with age within each study group and found that the frequency of only CD38expressing CD4 cells was positively associated with age in LTNPs $(p<.0001 ; r=0.50)$, while the $\mathrm{T}$ cell activation (HLA-DR- and/or CD38-expressing T cells) was not associated with age among the progressors. The HLA-DR ${ }^{+}$and CD38 ${ }^{+}$CD4 $\mathrm{T}$ cell frequencies were negatively associated with CD4 cell counts $(p<.0001$ and $p<.003$, respectively) (Fig. 1B). HLA-DR-expressing $\mathrm{CD}^{+}$and $\mathrm{CD} 8^{+} \mathrm{T}$ cells were associated with higher $\mathrm{pVL}(p<.001$ and $p<.01$, respectively) (Fig. 1C). Higher pVL was also found to be associated with $\mathrm{CD} 38^{+} \mathrm{CD} 8^{+} \mathrm{T}$ cells, close to the significance levels ( $p=.056 ; r=0.268)$. Furthermore, the frequencies of $\mathrm{T}$ cells coexpressing HLA-DR+CD38 were negatively associated with CD4 cell count (Fig. 1D) and positively with pVL (Fig. 1E).

The frequencies of both $\mathrm{CD} 4^{+}$and $\mathrm{CD} 8^{+} \mathrm{T}_{\mathrm{CM}}$ cells were significantly higher in LTNPs than progressors $(p<.0001)$ (Fig. 1F) and were associated with lower pVL $(p=.001$ and $p=.002$, respectively) (Fig. 1G), but not with CD4 count. In contrast, the frequencies of $\mathrm{CD}^{+} \mathrm{T}_{\mathrm{EM}}$ cells were significantly lower in LTNPs than in the progressors $(p<.0001)$ (Fig. 1F) and significantly associated with higher $\mathrm{pVL}$ $(p=.0009)$ (Fig. 1H). The $\mathrm{CD}^{+} \mathrm{T}_{\mathrm{EM}}$ frequencies were similar in progressors and LTNPs (Fig. 1F). We further assessed the association between the immune activation (activated $\mathrm{T}$ cells) and memory $\mathrm{T}$ cell population. A strong inverse correlation was observed between the activated $\mathrm{CD} 4^{+}$ and $\mathrm{CD}^{+} \mathrm{T}$ cells expressing HLA-DR+CD38 ${ }^{+}$with $\mathrm{CD}^{+}$ and $\mathrm{CD}^{+} \mathrm{T}_{\mathrm{CM}}$ (Fig. I, J respectively), while the $\mathrm{CD}^{+} \mathrm{T}_{\mathrm{EM}}$ frequency was positively associated with HLA-DR+CD4 ${ }^{+}$ $(r=0.594 ; p<.0001)$ and $\mathrm{CD}^{+} \mathrm{T}$ cells $(r=0.435 ; p=.0001)$.

Studies to identify correlates of immune mechanisms of HIV control are utmost essential for the development of new immune therapies as well as protection strategies. We, in this study, assessed the influence of immune activation on the memory $\mathrm{T}$ cell population that is important in mounting efficient recall responses. The study showed that the LTNPs exhibit lower T cell activation, higher $\mathrm{CD}^{+}$and $\mathrm{CD}^{+} \mathrm{T}_{\mathrm{CM}}$, and lower $\mathrm{T}_{\mathrm{EM}}$ populations compared to the chronic progressors. We also showed that the immune activation correlated with lower frequencies of $\mathrm{T}_{\mathrm{CM}}$ cells. A number of studies have shown that early immune activation causes damage to the immune system, which could result in chronic immune activation and further loss of immune function leading to disease progression. ${ }^{4,10,13,14,17}$ The CD38- and HLA-DR-expressing T cells are being used as a marker of immune hyperactivation and disease progression. ${ }^{4}$ These studies support our finding that LTNPs show less activation of $\mathrm{T}$ cells, indicating low inflammatory environment and thus preservation of CD4 count, and control over disease progression.

Strong $\mathrm{T}_{\mathrm{CM}}$ cell responses have shown to be associated with controlled disease progression. ${ }^{10,18-20}$ Our Indian LTNP 

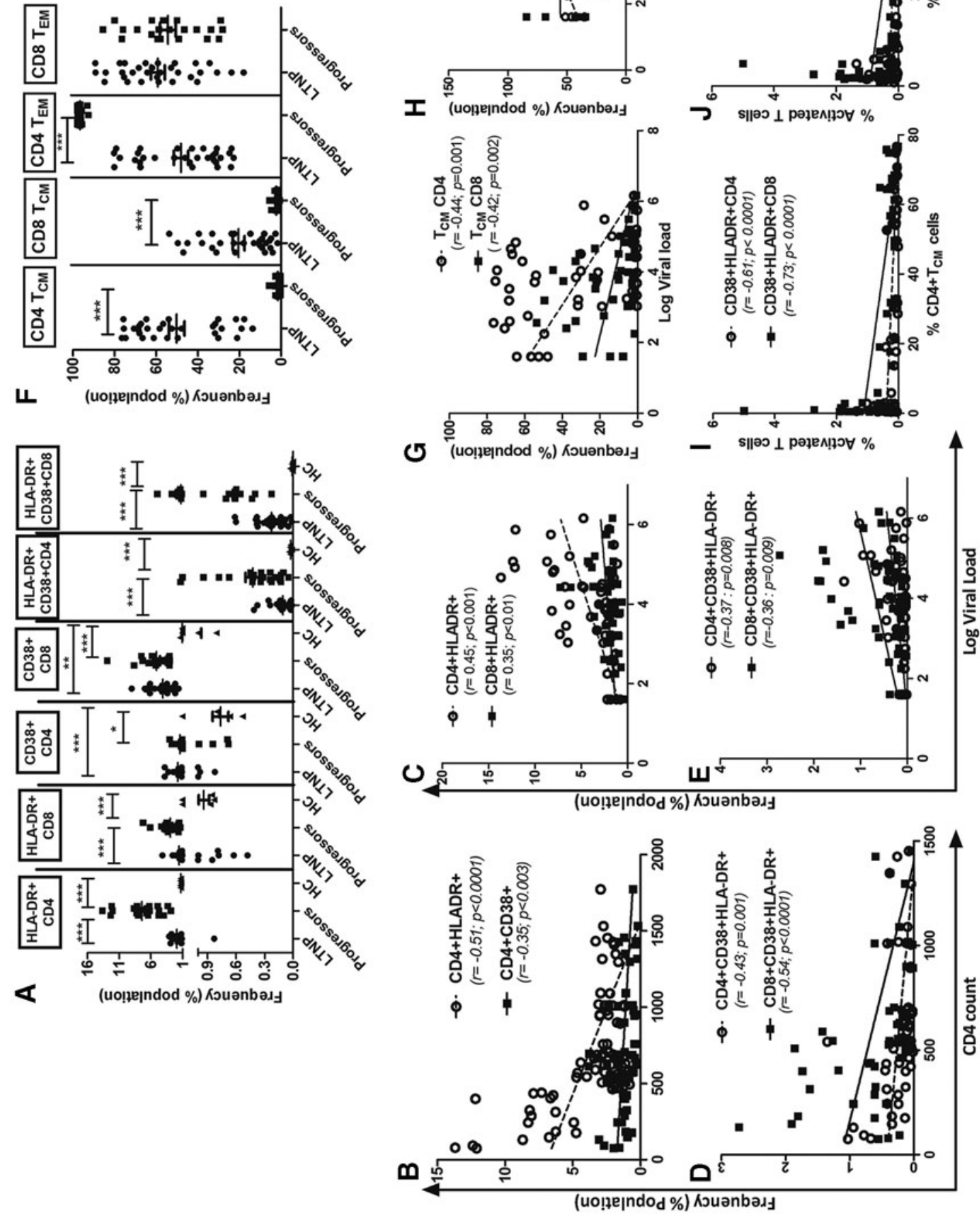

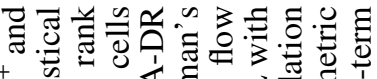

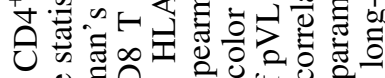

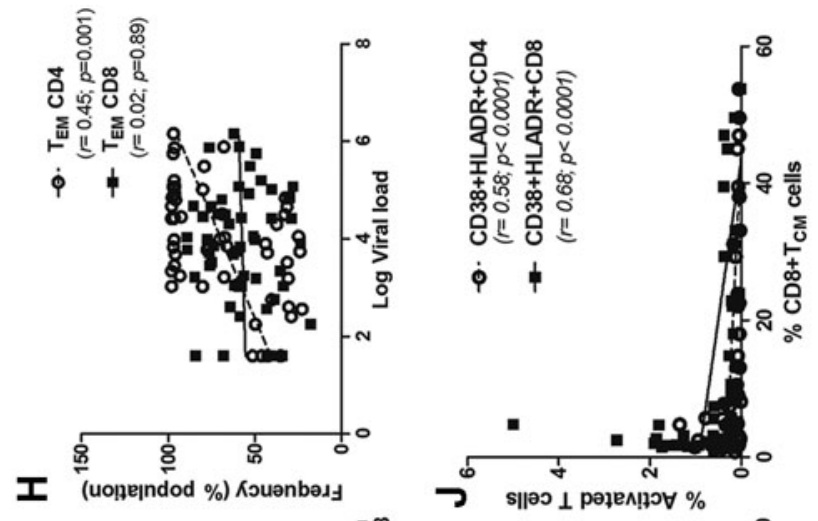

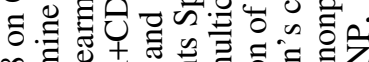

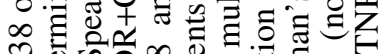

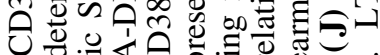

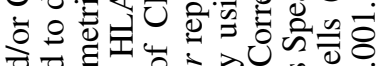

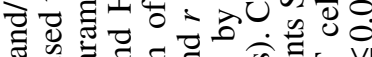

के สँ ส .

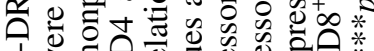

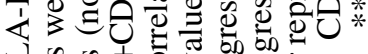

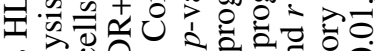

n

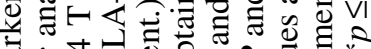

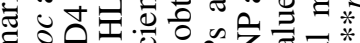

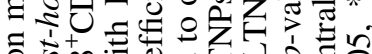

。ำ

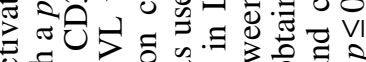

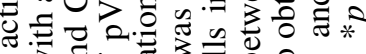

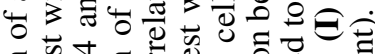

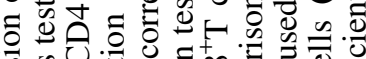
勿

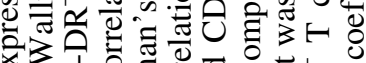

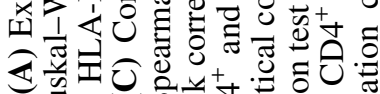

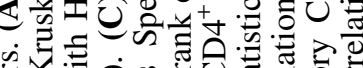

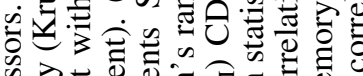

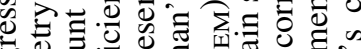
o。 元讨 ठी 은

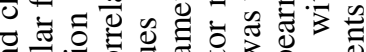
ส

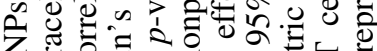

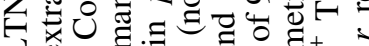

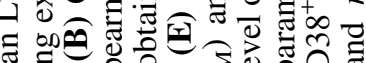

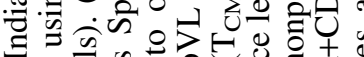

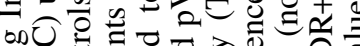

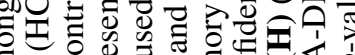

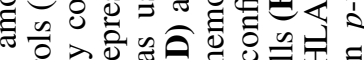

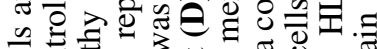
ठ

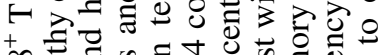
舟 U.

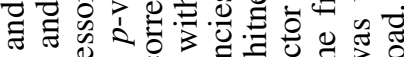

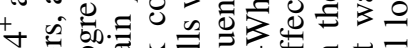

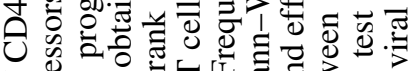
पे tho

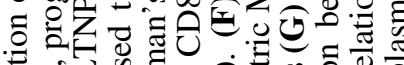

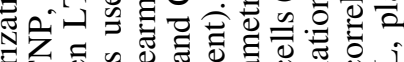

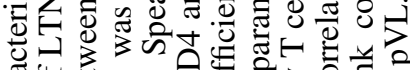
ซ్t 已゙ $+1.5$.




population also showed higher $\mathrm{T}_{\mathrm{CM}}$ population, supporting these observations. We also confirmed the previous finding of association of $\mathrm{T}_{\mathrm{EM}}$ with faster disease progression. ${ }^{6}$ The association of $\mathrm{T}_{\mathrm{EM}}$ frequencies with higher immune activation is also supported by previous observation in a mouse model where the immune activation showed a progressive conversion of naive $\mathrm{T}$ cells into effector memory cells. ${ }^{20}$

The increased immune activation is known to drive $\mathrm{T}$ cell differentiation leading to replicative senescence and immunodeficiencies. ${ }^{15,21}$ In HIV infection, this scenario in early course of HIV infection might result in loss of virus control leading to disease progression. During early HIV infection, higher $\mathrm{T}_{\mathrm{CM}}$ and $\mathrm{T}_{\mathrm{EM}}$ are associated with low and high viral load set point, respectively, indicating that early decrease in $\mathrm{T}_{\mathrm{CM}}$ cells might be contributing to high viral load and thus faster disease progression. ${ }^{6}$ In support to these previous observations, our study has shown that preserved $\mathrm{T}_{\mathrm{CM}}$ frequencies and lower immune activation are associated with controlled disease progression, although the causal relationship could not be established. It might be possible that lower immune activation in LTNPs is responsible for reduced perturbation in $\mathrm{T}$ cell differentiation and further apoptosis of $\mathrm{T}$ cells, resulting in preserved central memory $\mathrm{T}$ cells, which can effectively respond to the antigenic stimulus. This scenario might be collectively and/or partially contributing to the sustained CD4 counts in these patients even in the presence of high viral load. Indeed, the natural host of SIV infection, the sooty mangabey monkeys, do not develop AIDS despite high levels of virus replication and exhibit very low levels of immune activation. ${ }^{22}$

In summary, the low immune activation in HIV infection may contribute to preservation of memory cells and thus control over disease progression. The immune interventions to reduce the immune activation might be one of the useful anti-HIV strategies.

\section{Acknowledgments}

The authors would like to thank the study participants and the staff of clinic and immunology and virology laboratory of National AIDS Research Institute for their assistance.

\section{Financial Support}

This work was supported through institutional funding.

\section{Author Disclosure Statement}

No competing financial interests exist.

\section{References}

1. Taborda NA, Hernandez JC, Lajoie J, et al.: Short communication: Low expression of activation and inhibitory molecules on NK cells and CD4(+) T cells is associated with viral control. AIDS Res Hum Retroviruses 2015;31: 636-640.

2. Haas A, Zimmermann K, Oxenius A: Antigen-dependent and -independent mechanisms of $\mathrm{T}$ and $\mathrm{B}$ cell hyperactivation during chronic HIV-1 infection. J Virol 2011;85: 12102-12113.
3. Plaeger-Marshall S, Hultin P, Bertolli J, et al: Activation and differentiation antigens on $\mathrm{T}$ cells of healthy, at-risk, and HIV-infected children. J Acquir Immune Defic Syndr 1993;6:984-993.

4. Hazenberg MD, Otto SA, van Benthem BH, et al.: Persistent immune activation in HIV-1 infection is associated with progression to AIDS. AIDS 2003;17: 1881-1888.

5. Hazenberg MD, Stuart JW, Otto SA, et al.: T-cell division in human immunodeficiency virus (HIV)-1 infection is mainly due to immune activation: A longitudinal analysis in patients before and during highly active antiretroviral therapy (HAART). Blood 2000;95:249-255.

6. Burgers WA, Riou C, Mlotshwa M, et al.: Association of HIV-specific and total CD8+ T memory phenotypes in subtype C HIV-1 infection with viral set point. J Immunol 2009;182:4751-4761.

7. Okoye A, Meier-Schellersheim M, Brenchley JM, et al.: Progressive CD4+ central memory $\mathrm{T}$ cell decline results in CD4+ effector memory insufficiency and overt disease in chronic SIV infection. J Exp Med 2007;204:21712185.

8. McGary CS, Cervasi B, Chahroudi A, et al.: Increased stability and limited proliferation of CD4+ central memory $\mathrm{T}$ cells differentiate nonprogressive simian immunodeficiency virus (SIV) infection of sooty mangabeys from progressive SIV infection of rhesus macaques. J Virol 2014;88:4533-4542.

9. Mandalia S, Westrop SJ, Beck EJ, Nelson M, Gazzard BG, Imami N: Are long-term non-progressors very slow progressors? Insights from the Chelsea and Westminster HIV cohort, 1988-2010. PLoS One 2012;7:e29844.

10. Choudhary SK, Vrisekoop N, Jansen CA, et al.: Low immune activation despite high levels of pathogenic human immunodeficiency virus type 1 results in long-term asymptomatic disease. J Virol 2007;81:8838-8842.

11. Leon A, Leal L, Torres B, et al.: Association of microbial translocation biomarkers with clinical outcome in controllers HIV-infected patients. AIDS 2015;29:675-681.

12. Taborda NA, Catano JC, Delgado JC, Rugeles MT, Montoya CJ: Higher SLPI expression, lower immune activation, and increased frequency of immune cells in a cohort of Colombian HIV-1 controllers. J Acquir Immune Defic Syndr 2012;60:12-19.

13. Paiardini M, Muller-Trutwin M: HIV-associated chronic immune activation. Immunol Rev 2013;254:78-101.

14. Wilson CM, Ellenberg JH, Douglas SD, Moscicki AB, Holland CA: CD8+CD38+ T cells but not HIV type 1 RNA viral load predict $\mathrm{CD} 4+\mathrm{T}$ cell loss in a predominantly minority female HIV+ adolescent population. AIDS Res Hum Retroviruses 2004;20:263-269.

15. Papagno L, Spina CA, Marchant A, et al.: Immune activation and $\mathrm{CD} 8+\mathrm{T}$-cell differentiation towards senescence in HIV-1 infection. PLoS Biol 2004;2:E20.

16. Thakar MR, Bhonge LS, Lakhashe SK, et al.: Cytolytic T lymphocytes (CTLs) from HIV-1 subtype C-infected Indian patients recognize CTL epitopes from a conserved immunodominant region of HIV-1 Gag and Nef. J Infect Dis 2005;192:749-759.

17. Liovat AS, Jacquelin B, Ploquin MJ, Barre-Sinoussi F, Muller-Trutwin MC: African non human primates infected by SIV-Why don't they get sick? Lessons from studies on the early phase of non-pathogenic SIV infection. Curr HIV Res 2009;7:39-50. 
18. Potter SJ, Lacabaratz C, Lambotte O, et al.: Preserved central memory and activated effector memory CD4+ T-cell subsets in human immunodeficiency virus controllers: An ANRS EP36 study. J Virol 2007;81:1390413915.

19. Elrefaei M, McElroy MD, Preas CP, et al.: Central memory CD4+ $\mathrm{T}$ cell responses in chronic HIV infection are not restored by antiretroviral therapy. J Immunol 2004;173: 2184-2189.

20. Ndhlovu ZM, Proudfoot J, Cesa K, et al.: Elite controllers with low to absent effector CD8+ T cell responses maintain highly functional, broadly directed central memory responses. J Virol 2012;86:6959-6969.

21. Tesselaar K, Arens R, van Schijndel GM, et al.: Lethal T cell immunodeficiency induced by chronic costimulation via CD27-CD70 interactions. Nat Immunol 2003;4: 49-54.
22. Silvestri G, Sodora DL, Koup RA, et al: Nonpathogenic SIV infection of sooty mangabeys is characterized by limited bystander immunopathology despite chronic highlevel viremia. Immunity 2003;18:441-452.

Address correspondence to: Madhuri Thakar Department of Immunology and Serology National AIDS Research Institute MIDC

Plot No. 73, G-Block Bhosari

Pune 411026

Maharashtra India

E-mail: mthakar@icmr.org.in; mthakar@nariindia.org 